\title{
Analysis of the dynamics of an active control of the surface potential in metal oxide gas sensors
}

\author{
Oscar Monge-Villora ${ }^{\mathrm{a}, *}$, Manuel Dominguez-Pumar ${ }^{\mathrm{a}, *}$, Josep M. Olmº,* \\ ${ }^{a}$ Micro and Nano Technologies Group, Universitat Politècnica de Catalunya, Barcelona, \\ Spain \\ ${ }^{b}$ Department of Mathematics $\&$ Institute of Industrial and Control Engineering, \\ Universitat Politècnica de Catalunya, Barcelona, Spain
}

\begin{abstract}
Gas sensing is nowadays a key actor in pollution observation and detection of chemical toxic agents or explosives. All these applications require the shortest possible time response. Very recently, a control of the surface potential in gas sensors based on metal oxides has experimentally shown to dramatically improve the time response of metal-oxide gas sensors. The proposed control is inspired in sigma-delta modulators. This paper aims at studying the resulting dynamics in the sensor from a theoretical point of view. Using state space models, it is shown how the state variables, namely the concentrations of ionized species in the sensing layer, evolve with time in open and closed loop configuration. This analysis studies how it is possible to alter the dynamics of the overall system, while at the same time keeping some important characteristics of sigma-delta modulators, such as quantization noise-shaping. Numerical simulations validate the obtained results.
\end{abstract}

Keywords: Chemical sensors, surface potential, sliding mode control.

\section{Introduction}

Metal-oxide (MOX) gas sensors are used in a wide field of different applications requiring gas sensing. Among the most prominent ones we find

\footnotetext{
*Corresponding author: Oscar Monge-Villora

Email addresses: oscar.monge@upc.edu (Oscar Monge-Villora), manuel.dominguez@upc.edu (Manuel Dominguez-Pumar), josep.olm@upc.edu (Josep M. Olm)
} 
air quality monitoring, detection of gas leaks in pipes, detection of hazardous gases, detection of smouldering underground fires, among many others. [1, 2, 3]. MOX gas sensors present many advantages such as: high sensitivity, low-cost production, small size, low power consumption and high compatibility with semiconductor manufacturing industry $[4,5,6,7,8]$.

The typical operation of MOX gas sensors consists on monitoring the resistivity of a sensing layer, made of a semiconductor metal oxide, using interdigitated electrodes. Many different materials, such as $\mathrm{SnO}_{2}, \mathrm{WO}_{3}$ or $\mathrm{ZnO}$, and deposition/growth techniques have been developed to conform the sensing layers. The sensing layers can be thick, thin or nanostructured (nanowires, nanorods, nanoparticles, etc.) and can even be decorated with nanoparticles made of $\mathrm{Au}$, Co or Pt, which act as catalysts.

The sensor is usually operated at constant temperature, within some specific temperature ranges which depend on the materials of the sensing layer, and are normally well above room temperature $\left(100^{\circ} \mathrm{C}-400^{\circ} \mathrm{C}\right)$. In order to reach and maintain this operating temperature, a heater is usually embedded in the structure of the sensor.

Many performance aspects of these sensors are being continuously studied and improved. Nowadays, for example, in order to reduce power consumption, it is usual to place the sensing layers and associated heaters on top of silicon microhotplates, conforming what is called a Microelectromechanical System (MEMS) [9].

The conductance of the sensing layer is basically determined by temperature and by some electronic molecular mechanisms based on the adsorption and ionization processes of the gases present in the surrounding atmosphere [10]. The adsorbed molecules create some localized energy levels that appear in the forbidden gap of the band diagram, known as extrinsic surface states. These states, added to the inherent intrinsic surface states, are able to trap or release electrons at the conduction band of the material, modifying the conductance response [11]. Usually, rate equation models are used to describe the time evolution of chemisorbed and ionized surface states $[12,13,14,15,16,17]$. The balance of charge in the charge space regions of the semiconductor determines the surface potential, which, together with temperature, determine the conductivity of the sensing layer.

Extensive work has been made on how to improve the sensor response, understood as a system. In some cases, temperature modulations are used to generate some dynamical patterns of the conductivity, useful to recognize the species present on the gas mixture. Other proposals are based on digital 
signal processing of the sensor signals as neural networks [18], or reservoir computing algorithms [19]. We can find some examples in the literature, [20], that propose specific methodologies to create and analyze dynamic patterns. Most works, though, have focused on improving sensor performance using new materials and fabrication techniques, such as Metal-Organic-Frameworks (MOFs) [21], 3D hierarchical nanostructures [22], or poly(N-vinylcarbazole) grafted multi-walled carbon nanotubes [23].

Very recently, a control of the surface potential for MOX gas sensors has been proposed for the first time [24, 25]. This control is based on sigmadelta modulation, understood as a way of enforcing a sliding mode control $[26,27,28,29]$ on the state variables of the sensor [30]. In order to do this, a feedback loop using temperature modulation is used to operate the sensor aiming at achieving a constant surface potential.

The objective of this paper is the analysis of the time evolution of the state variables of the sensor (concentrations of adsorbed and ionized species) and their equilibrium points in closed and open loop configuration. For the first time, the dynamics is studied using sliding mode analysis and similar techniques to those proposed in [31] to control charge trapping in dielectrics. In order to check the improvements of the proposed control method, we use models available in the literature describing the chemical behavior of the sensing layer when exposed to some reducing or oxidising species. These models are based on rate equations of adsorption and ionization processes. These aspects are briefly described in Section 3. Before that, in Section 2, the sliding mode-based control method is described and linked with the theory explaining how the conductivity of the sensing layer depends on the surface potential. In Sections 4 and 5 we analyze the open and closed loop dynamics, respectively, in the latter using the theory of sliding mode control. Numerical results are shown in Section 6, comparing the open and closed loop responses of the sensor. Finally, conclusions and some suggestions for further research are drawn in Section 7.

\section{Sigma-delta control of the surface potential: an overview}

\subsection{Control method}

As it has been mentioned in Section 1, the objective of the control method is to set a constant surface potential value in the sensing layer. For most 
metal oxides, the conductivity follows this expression [24]:

$$
G=G_{0}(T) \exp \left(-\frac{q V_{s}}{k T}\right)
$$

where $G_{0}(T)$ is a factor depending on the temperature, $T, q$ is the charge of an electron, $V_{s}$ is the surface potential and $k$ is the Boltzmann constant. Now, it is obvious from this expression that keeping constant the conductivity of the sensing layer, $G$, measured at constant temperature, is equivalent to enforce a constant surface potential in the sensing layer.

The proposed surface potential control method applies suitable temperature waveforms that end with the same temperature value. By doing this, it is possible to monitor periodically the conductivity of the sensing layer, measured at constant temperature. At the same time, changing the sequence of temperature waveforms allows modulating the average temperature of the sensing layer. This is the actuation mechanism used to obtain the desired values of the surface potential.

The control method is a discrete-time feedback control scheme inspired on sigma-delta modulation, using a 1-bit quantizer. In the implementation of [24, 25], during each sampling period, two temperature profiles can be applied, denoted as $T_{\mathrm{BIT} 0}$ and $T_{\mathrm{BIT} 1}$, see Figure 1 . Both waveforms make use of two different temperatures, namely $T_{l}$ and $T_{h}$, with $T_{l}<T_{h}$, and are defined as:

$$
\begin{aligned}
& T_{\mathrm{BIT} 0}(t)= \begin{cases}T_{l}, & t \in\left[0,(1-\delta) T_{s}\right) \\
T_{h}, & t \in\left[(1-\delta) T_{s}, T_{s}\right)\end{cases} \\
& T_{\mathrm{BIT} 1}(t)= \begin{cases}T_{l}, & t \in\left[0, \delta T_{s}\right) \\
T_{h}, & t \in\left[\delta T_{s}, T_{s}\right)\end{cases}
\end{aligned}
$$

Following the conventional sigma-delta modulation scheme, depending on whether the resistivity of the layer is above (or below) a desired target value, a $T_{\mathrm{BIT0}}\left(T_{\mathrm{BIT} 1}\right)$ temperature is applied during the next sampling period. An output bitstream is formed following the sequence of temperature waveforms generated by the control $\left(b_{n}=1\right.$ when $T_{\mathrm{BIT} 1}$ is applied during $\left[n T_{S},(n+1) T_{S}\right]$ and $b_{n}=0$ when $T_{\text {BIT0 }}$ is applied during $\left.\left[n T_{S},(n+1) T_{S}\right]\right)$. The output of the sensor, working in closed loop configuration now, is either: 
- the moving average of $(\mathrm{N}+1)$ samples of the generated bitstream: $\bar{b}_{n}=$ $\sum_{k=n-N}^{n} b_{k} /(N+1)$,

- or the average temperature generated by the control:

$$
\bar{T}_{n}=\sum_{k=n-N}^{n}\left(b_{n} \bar{T}_{1}+\left(1-b_{n}\right) \bar{T}_{0}\right) /(N+1)
$$

where $\bar{T}_{0}$ and $\bar{T}_{1}$ are the average temperature of the $T_{\mathrm{BIT} 0}$ and $T_{\mathrm{BIT} 1}$ waveforms respectively.

Now, considering an oxidizing atmosphere, when the resistivity of the sensor is below the desired value, the control tends to increase the average temperature thus enhancing the oxidation processes, which tend to increase resistivity.

The dynamics of the control loop presents two stages. During the first stage, the control only applies one of the two actuation waveforms until it reaches the desired surface potential value; in sliding mode control this is known as the reaching phase [26]. After that, it enters a second phase in which the control applies a convenient sequence of $T_{\mathrm{BIT} 0}$ and $T_{\mathrm{BIT} 1}$ to keep the conductance of the sensor around its target value.

In next subsection, we analyze one of the possible conduction mechanisms in the sensing layer allowing to link measurements (conductance) with the state variables (concentration of species).

\subsection{Potential barrier theory, surface states and conductivity}

Potential barrier theory is one of the most common theories describing conduction in MOX layers. It relates the surface state trapping models [11, 32 in the layer, with its conductivity. Surface states are energy levels on the band diagram. These can be caused by the adsorption of gas molecules (extrinsic surface states) or by impurities, oxygen vacancies, etc., which are inherent to the material (intrinsic surface states). They can trap/detrap free electrons, or holes, thus becoming ionized surface states (negatively or positively charged), or neutral.

For an n-type metal oxide, the total density of charged surface states, $N_{s}$, is related with the surface potential, $V_{s}$, in a quadratic form [11]:

$$
V_{s}=\frac{q N_{s}^{2}}{2 \epsilon N_{d}}
$$




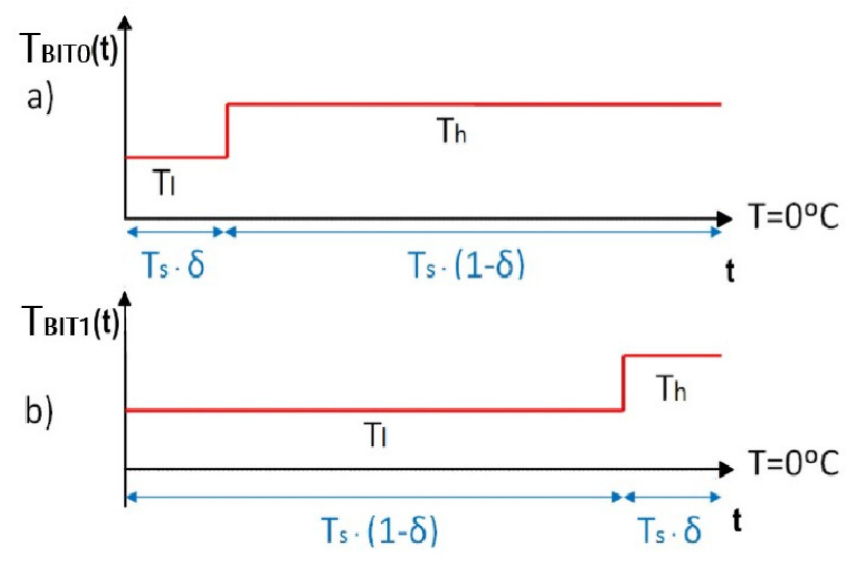

Figure 1: Temperature actuation waveforms. (a) $T_{B I T 1}$ applies $T_{l}$ for a short time $\delta T_{S}$, followed by $T_{h}$ for a time $(1-\delta) T_{S}$. (b) In $T_{B I T 0}, T_{l}$ is applied during $(1-\delta) T_{S}$, then $T_{h}$.

where $\epsilon$ is the permittivity, $N_{d}$ is the donors' impurities density in the material, and $q$ is the electron charge.

Therefore, the expression of the conductivity, (1), becomes [24]:

$$
G\left(T, V_{s}\right)=G_{0}(T) \exp \left(-\frac{q^{2} N_{s}^{2}}{2 k \epsilon N_{d} T}\right),
$$

$G_{0}(T)$ being a pre-exponential factor. It must be noted that different conduction mechanisms present different temperature dependences in the preexponential factor, but follow the same expression.

Therefore, controlling: (i) the conductivity, $G$, measured at constant temperature, or (ii) the surface potential, $V_{s}$, or (iii) the total density of charged surface states, $N_{s}$, are equivalent processes.

\section{Rate equations models}

In this section we describe briefly the rate equation models chosen from the literature $[33,11]$ for the analysis.

\subsection{Model 1: sensing of $\mathrm{NO}_{2}$}

The first model [33] used to test the sliding control mode is based on exposing the chemical sensor to an oxidising species, $\mathrm{NO}_{2}$. The chemical 


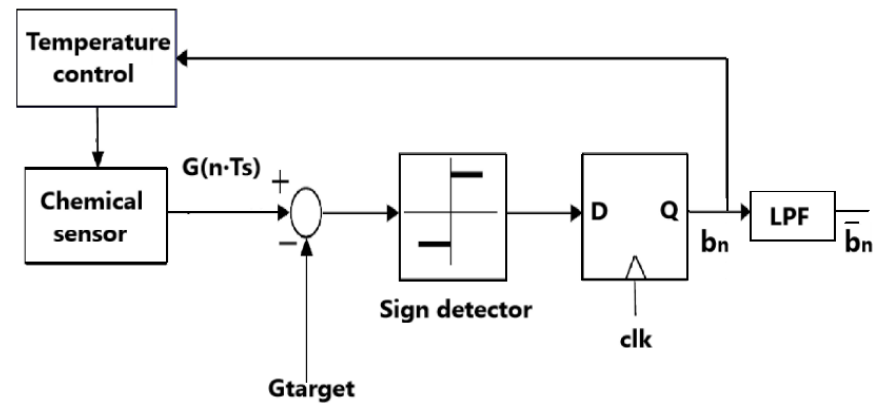

Figure 2: Closed loop system scheme proposed in [24, 25]

reactions involved are:

$$
\begin{aligned}
& n \mathrm{~S}+\frac{1}{2} \mathrm{O}_{2}+\mathrm{e} \underset{k_{-1}}{\stackrel{k_{1}}{\rightleftharpoons}}\left(\mathrm{S}-\mathrm{O}^{-}\right)+(n-1) \mathrm{S} \\
& n \mathrm{~S}+\mathrm{NO}_{2}+\mathrm{e} \underset{k_{2}}{\stackrel{k_{-2}}{\rightleftharpoons}}\left(\mathrm{S}_{-} \mathrm{NO}_{2}^{-}\right)+(n-1) \mathrm{S} .
\end{aligned}
$$

Following the notation of [33], $\mathrm{S}$ is the representation of a free adsorbing site, $n$ is the total number of free adsorbing sites, $\left(\mathrm{S}_{-} \mathrm{O}^{-}\right)$and $\left(\mathrm{S}_{-} \mathrm{NO}_{2}^{-}\right)$are the number of ionized surface states and $k_{i}$ are the rate reaction parameters. The rate equations extracted from the chemical reactions are the following:

$$
\begin{aligned}
& \frac{d\left[\mathrm{~S}-\mathrm{O}^{-}\right]}{d t}=k_{1}\left[\mathrm{O}_{2}\right]^{1 / 2}\left(\mathrm{~S}_{0}-\left[\mathrm{S}-\mathrm{O}^{-}\right]-\left[\mathrm{S}^{-\mathrm{NO}_{2}^{-}}\right]\right)-k_{-1}\left[\mathrm{~S}-\mathrm{O}^{-}\right] \\
& \frac{d\left[\mathrm{~S}^{-} \mathrm{NO}_{2}^{-}\right]}{d t}=k_{2}\left[\mathrm{NO}_{2}\right]\left(\mathrm{S}_{0}-\left[\mathrm{S}-\mathrm{NO}_{2}^{-}\right]\right)-k_{-2}\left[\mathrm{~S}_{-} \mathrm{NO}_{2}^{-}\right]
\end{aligned}
$$

where $\mathrm{S}_{0}$ is constant and represents the maximum concentration of extrinsic surface states. Now, $\left[\mathrm{S}_{-} \mathrm{O}^{-}\right]$and $\left[\mathrm{S}^{-} \mathrm{NO}_{2}^{-}\right]$are the densities of ionized species, and $\left[\mathrm{O}_{2}\right]$ and $\left[\mathrm{NO}_{2}\right]$ are the external gas concentrations. Typically $\left[\mathrm{O}_{2}\right]$ is constant while, in experiments, $\left[\mathrm{NO}_{2}\right]$ changes with time. The total density of charged surface states is:

$$
N_{s}=\left[\mathrm{S}-\mathrm{O}^{-}\right]+\left[\mathrm{S}-\mathrm{NO}_{2}^{-}\right] .
$$


Table 1: Parameter values for Model 1 [33]

\begin{tabular}{lcccr}
$k_{10}\left(\mathrm{~s}^{-1} \mathrm{ppm}^{-1 / 2}\right)$ & $k_{-10}\left(\mathrm{~s}^{-1}\right)$ & $k_{20}\left(\mathrm{~s}^{-1}\right)$ & $k_{-20}\left(\mathrm{~s}^{-1}\right)$ & $N_{d}\left(\mathrm{~m}^{-3}\right)$ \\
\hline $1.4 \cdot 10^{-5}$ & $1.1 \cdot 10^{-1}$ & $2.7 \cdot 10^{-5}$ & $1.8 \cdot 10^{-3}$ & $1.4 \cdot 10^{24}$ \\
\hline & & & & \\
$S_{0}\left(\mathrm{ppm} \cdot \mathrm{m}^{-2}\right)$ & $E_{1}(\mathrm{~J} / \mathrm{mol})$ & $E_{-1}(\mathrm{~J} / \mathrm{mol})$ & $E_{2}(\mathrm{~J} / \mathrm{mol})$ & $E_{-2}(\mathrm{~J} / \mathrm{mol})$ \\
\hline $1.3 \cdot 10^{17}$ & $5.1 \cdot 10^{5}$ & $1.2 \cdot 10^{4}$ & $5.9 \cdot 10^{4}$ & $0.7 \cdot 10^{2}$
\end{tabular}

The rate reaction parameters are governed by the Arrhenius law, which describes their exponential dependence on temperature:

$$
k_{x}=k_{x 0} \exp \left(\frac{-E_{x}}{k T}\right)=k_{x 0} \exp \left(\frac{-\lambda_{x}}{T}\right)
$$

where $k_{x 0}$ is a constant pre-exponential factor, $E_{x}$ the activation energy and $\lambda_{x}$ a normalized activation energy. The values of the different $k_{x 0}$ and $E_{x}$ constants used in [33] are specified in Table 1. At constant temperature, and constant gas concentrations, (5),(6) is a coupled linear system.

\subsection{Model 2: sensing of $C O$}

The second model used in this work describes the adsorption and ionization of a reducing gas, CO, in synthetic air. The chemical reactions involved are:

$$
\begin{aligned}
& \mathrm{CO}+\mathrm{S}_{\mathrm{CO}} \underset{k_{-\mathrm{CO}}}{\stackrel{k_{\mathrm{CO}}}{\rightleftharpoons}}\left(\mathrm{CO}_{-} \mathrm{S}_{\mathrm{CO}}\right) \quad(\mathrm{CO} \text { adsorption }) \\
& \left.\left(\mathrm{CO}-\mathrm{S}_{\mathrm{CO}}\right) \underset{k_{-\mathrm{CO}^{+}}}{\stackrel{k_{\mathrm{CO}}}{\rightleftharpoons}}\left(\mathrm{CO}^{+}{ }_{-} \mathrm{S}_{\mathrm{CO}}\right)+\mathrm{e}^{-} \text {(CO ionization }\right) \\
& \frac{1}{2} \mathrm{O}_{2}+\mathrm{S}_{\mathrm{O}} \underset{k_{-\mathrm{O}}}{\stackrel{k_{\mathrm{O}}}{\rightleftharpoons}}\left(\mathrm{O}-\mathrm{S}_{\mathrm{O}}\right) \quad(\mathrm{O} \text { adsorption }) \\
& \left(\mathrm{O}-\mathrm{S}_{\mathrm{O}}\right)+\mathrm{e}^{-\frac{k_{\mathrm{O}^{-}}}{\rightleftharpoons}}\left(\mathrm{O}^{-}-\mathrm{S}_{\mathrm{O}}\right) \quad(\mathrm{O} \text { ionization }) \\
& \left(\mathrm{O}^{-}-\mathrm{S}_{\mathrm{O}}\right)+\mathrm{CO} \stackrel{k_{\mathrm{CO}_{2}}}{\rightleftharpoons} \mathrm{CO}_{2}+\mathrm{S}_{\mathrm{O}}+\mathrm{e}^{-} \text {(CO oxidation) }
\end{aligned}
$$

The total density of charged surface states in this case is:

$$
N_{s}=N_{s i}+\left[\mathrm{O}^{-}-\mathrm{S}_{\mathrm{O}}\right]-\left[\mathrm{CO}^{+}-\mathrm{S}_{\mathrm{CO}}\right],
$$

where $N_{s i},\left[\mathrm{O}^{-}-\mathrm{S}_{\mathrm{O}}\right]$ and $\left[\mathrm{CO}^{+}-\mathrm{S}_{\mathrm{CO}}\right]$ are the density of ionized intrinsic surface states and the densities of ionized extrinsic surface states (originated by 
oxygen molecules or carbon monoxide molecules), respectively. We assume that adsorption is a dynamical process much faster than the process associated with the ionization of species [11]. This means that the relevant state variables in the system are the concentration of ionized species in the sensing layer.

The dynamics of the density of intrinsic and extrinsic states are described by:

$$
\begin{aligned}
& \frac{d N_{s i}}{d t}=k_{i} n_{s}\left(N_{i}-N_{s i}\right)-k_{-i} N_{s i} \\
& \frac{d\left[\mathrm{CO}^{+}{ }_{-} \mathrm{S}_{\mathrm{CO}}\right]}{d t}=k_{\mathrm{CO}^{+}}\left[\mathrm{CO}-\mathrm{S}_{\mathrm{CO}}\right]_{0}-k_{-\mathrm{CO}^{+}}\left[\mathrm{CO}^{+}{ }_{-} \mathrm{S}_{\mathrm{CO}}\right] n_{s} \\
& \frac{d\left[\mathrm{O}^{+}{ }_{-} \mathrm{S}_{\mathrm{O}}\right]}{d t}=k_{\mathrm{O}^{-}}\left[\mathrm{O}-\mathrm{S}_{\mathrm{O}}\right]_{0} n_{s}-k_{-\mathrm{O}^{-}}\left[\mathrm{O}^{-}{ }_{-} \mathrm{S}_{\mathrm{O}}\right]-k_{\mathrm{CO}_{2}}[\mathrm{CO}]\left[\mathrm{O}^{-}{ }_{-} \mathrm{S}_{\mathrm{O}}\right]
\end{aligned}
$$

where $N_{i}$ is the total intrinsic density of states (ionized plus non-ionized) and $k_{Y}, Y= \pm i, \mathrm{O}^{-}, \mathrm{CO}^{+}, \mathrm{CO}_{2}$, are the rate reaction constants. In turn, $\left[\mathrm{CO}-\mathrm{S}_{\mathrm{CO}}\right]_{0}$ and $\left[\mathrm{O}-\mathrm{S}_{\mathrm{O}}\right]_{0}$ are the equilibrium densities of extrinsic surface states obtained in the adsorption process, which depend on the external CO concentration, $[\mathrm{CO}]$.

It is important to take into account that (9)-(11) is a nonlinear differential system of three equations coupled by the nonlinear term $n_{s}$, the density of electrons in the conduction band. This term depends on the total ionized density of states, $N_{s}$, following this expression [11] :

$$
n_{s}=N_{d} \exp \left(-\frac{q V_{s}}{k T}\right)=N_{d} \exp \left(-\frac{q^{2} N_{s}^{2}}{2 k \epsilon N_{d} T}\right) .
$$

The next step is to normalize the state variables [34]:

$$
[X]^{\prime}=\frac{q}{\sqrt{2 \epsilon N_{d} k}}[X]=\alpha[X], \text { where } \alpha=\frac{q}{\sqrt{2 \epsilon N_{d} k}} .
$$


Table 2: Parameter values for Model $2\left(\mathrm{O}_{2}\right.$ and $\mathrm{CO}_{2}$ adsorption and ionization [34] $)$

\begin{tabular}{ccccc}
$A_{\mathrm{CO}^{+}}\left(\mathrm{K}^{1 / 2} / \mathrm{s}\right)$ & $B_{\mathrm{CO}^{+}}(1 / \mathrm{s})$ & $\hat{C}_{\mathrm{CO}^{+}}$ & $\lambda_{\mathrm{CO}^{+}}(\mathrm{K})$ & $\lambda_{-\mathrm{CO}^{+}}(\mathrm{K})$ \\
\hline 1130 & 91.9 & $69 \cdot 10^{6}$ & 2500 & 573 \\
\hline$\lambda_{\mathrm{CO}^{+}}^{\prime}(\mathrm{K})$ & $A_{\mathrm{O}^{-}}\left(\mathrm{K}^{1 / 2} / \mathrm{s}\right)$ & $B_{\mathrm{O}^{-}}(1 / \mathrm{s})$ & $\lambda_{\mathrm{O}^{-}}(\mathrm{K})$ & $\lambda_{-\mathrm{O}^{-}}(\mathrm{K})$ \\
\hline 3990 & 4020 & 0.0110 & 677 & 1620
\end{tabular}

Table 3: Parameter values for Model 2 (Ionization of intrinsic states and CO oxidation)

\begin{tabular}{cccc}
$A_{i}(1 / \mathrm{s})$ & $B_{i}(1 / \mathrm{s})$ & $\lambda_{i}(\mathrm{~K}) ;$ & $\lambda_{-i}(\mathrm{~K})$ \\
\hline 2252 & 0.443 & 2050 & 3720 \\
\hline & & & \\
$N_{i}^{\prime}\left(\mathrm{K}^{1 / 2}\right)$ & $G_{0}^{*}(\mathrm{~S})$ & $\hat{A}_{\mathrm{CO}_{2}}\left(\mathrm{~K}^{1 / 2} / \mathrm{s}\right)$ & $\lambda_{\mathrm{CO}_{2}}(\mathrm{~K})$ \\
\hline 111 & 10500 & $2.13 \cdot 10^{-6}$ & 786
\end{tabular}

Applying this normalization and regrouping terms we have that:

$$
\begin{aligned}
& \frac{d N_{s i}^{\prime}}{d t}=A_{i} e^{-\frac{\lambda_{i}+N_{s}^{\prime 2}}{T}}\left(N_{i}^{\prime}-N_{s i}^{\prime}\right)-B_{i} e^{-\frac{\lambda_{-i}}{T}} N_{s i}^{\prime}, \\
& \frac{d\left[\mathrm{CO}^{+}-\mathrm{S}_{\mathrm{CO}}\right]^{\prime}}{d t}=\frac{A_{\mathrm{CO}^{+}} e^{-\frac{\lambda_{\mathrm{CO}+}}{T}}}{1+\frac{\hat{C}_{\mathrm{CO}+}}{[\mathrm{CO}]} e^{-\frac{\lambda_{\mathrm{CO}+}^{\prime}}{T}}}-B_{\mathrm{CO}^{+}} e^{-\frac{\lambda_{-} \mathrm{CO}^{+}+N_{s}^{\prime 2}}{T}}\left[\mathrm{CO}^{+}-\mathrm{S}_{\mathrm{CO}}\right]^{\prime} \\
& \frac{d\left[\mathrm{O}^{\prime}-\mathrm{S}_{\mathrm{O}}\right]^{\prime}}{d t}=A_{\mathrm{O}^{-}} e^{-\frac{\lambda_{\mathrm{O}^{-}+N_{s}^{\prime}}}{T}}-B_{\mathrm{O}^{-}} e^{-\frac{\lambda_{-}-\mathrm{O}^{-}}{T}}\left[\mathrm{O}^{-}-\mathrm{S}_{\mathrm{O}}\right]^{\prime}+ \\
& -\hat{A}_{\mathrm{CO}_{2}} e^{-\frac{\lambda_{\mathrm{CO}_{2}}^{T}}{T}}[\mathrm{CO}]\left[\mathrm{O}^{-}-\mathrm{S}_{\mathrm{O}}\right]^{\prime}
\end{aligned}
$$

where $A_{i}, B_{i}, \lambda_{i}, \lambda_{-i}, N_{i}^{\prime}, A_{\mathrm{CO}^{+}}, B_{\mathrm{CO}^{+}}, \hat{C}_{\mathrm{CO}^{+}}, \lambda_{\mathrm{CO}^{+}}, \lambda_{-\mathrm{CO}^{+}}, \lambda_{\mathrm{CO}^{+}}^{\prime}, A_{\mathrm{O}^{-}}, B_{\mathrm{O}^{-}}$, $\lambda_{\mathrm{O}^{-}}, \lambda_{-\mathrm{O}^{-}}, \hat{A}_{\mathrm{CO}_{2}}, \lambda_{\mathrm{CO}_{2}}$ are constants with values specified in Tables 2 and 3. This normalization, applied to the conductivity (4), yields:

$$
G\left(T, N_{s}^{\prime}\right)=G_{0}(T) \exp \left(-\frac{N_{s}^{\prime 2}}{T}\right)
$$

\subsection{Unified state-space representation of the chemical models}

The dynamical models (5),(6) and (12)-(14) allow a unified state space representation in the usual control systems form, namely,

$$
\dot{x}=f(x, u, \mu),
$$


where $x$ is the state vector, $u:=T(t)$ is the control variable, and $\mu$ stands for the concentration of the gas to be sensed, which in this case is taken as a piecewise constant parameter. Specifically, for Model 1 it is

$$
x=\left(\left[\mathrm{S}-\mathrm{O}^{-}\right],\left[\mathrm{S}-\mathrm{NO}_{2}^{-}\right]\right)^{\top}, \quad \mu=\left[\mathrm{NO}_{2}\right],
$$

so (5),(6) can be rewritten as:

$$
\begin{aligned}
& \dot{x}_{1}=-\left(a_{11}(u)+a_{12}(u)\right) x_{1}-a_{11}(u) x_{2}+a_{11}(u) \mathrm{S}_{0}, \\
& \dot{x}_{2}=-\left(\mu a_{21}(u)+a_{22}(u)\right) x_{2}+\mu a_{21}(u) \mathrm{S}_{0},
\end{aligned}
$$

with

$$
\begin{aligned}
& a_{11}(u)=k_{-10} e^{-\frac{\lambda_{-1}}{u}}, a_{12}(u)=\left[\mathrm{O}_{2}\right]^{1 / 2} k_{10} e^{-\frac{\lambda_{1}}{u}} \\
& a_{21}(u)=k_{20} e^{-\frac{\lambda_{2}}{u}}, a_{22}(u)=k_{-20} e^{-\frac{\lambda_{-2}}{u}} .
\end{aligned}
$$

In turn, for Model 2 it is

$$
x=\left(N_{s i}^{\prime},\left[\mathrm{CO}^{+}-\mathrm{S}_{\mathrm{CO}}\right]^{\prime},\left[\mathrm{O}^{-}-\mathrm{S}_{\mathrm{O}}\right]^{\prime}\right)^{\top}, \quad \mu=[\mathrm{CO}],
$$

and (12)-(14) become:

$$
\begin{aligned}
& \dot{x}_{1}=a_{11}(u) e^{-\frac{\left(x_{1}-x_{2}+x_{3}\right)^{2}}{u}}\left(N_{i}^{\prime}-x_{1}\right)-a_{12}(u) x_{1}, \\
& \dot{x}_{2}=a_{21}(u, \mu)-a_{22}(u) e^{-\frac{\left(x_{1}-x_{2}+x_{3}\right)^{2}}{u}} x_{2}, \\
& \dot{x}_{3}=a_{31}(u, \mu) e^{-\frac{\left(x_{1}-x_{2}+x_{3}\right)^{2}}{u}}-a_{32}(u, \mu) x_{3},
\end{aligned}
$$

with

$$
\begin{aligned}
& a_{11}(u)=A_{i} e^{-\frac{\lambda_{i}}{u}}, a_{12}(u)=-B_{i} e^{-\frac{\lambda_{-i}}{u}}, a_{21}(u, \mu)=\frac{\mu A_{\mathrm{CO}^{+}} e^{-\frac{\lambda_{\mathrm{CO}}+}{u}}}{\mu+\hat{C}_{\mathrm{CO}^{+}} e^{-\frac{\lambda_{\mathrm{CO}+}^{\prime}}{u}}}, \\
& a_{22}(u)=B_{\mathrm{CO}^{+}} e^{-\frac{\lambda_{-} \mathrm{CO}^{+}}{u}}, a_{31}(u)=A_{\mathrm{O}^{-}} e^{-\frac{\lambda_{\mathrm{O}^{-}}}{u}}, \\
& a_{32}(u, \mu)=B_{\mathrm{O}^{-}} e^{-\frac{\lambda_{-}-}{u}}+\mu \hat{A}_{\mathrm{CO}_{2}} e^{-\frac{\lambda_{\mathrm{CO}_{2}}}{u}} \text {. }
\end{aligned}
$$

Notice that it follows immediately from Tables 1, 2 and 3 that $a_{i j}>0$, for all $a_{i j}$ in (19),(20) and (24)-(26). 


\section{Open loop analysis}

In this Section the system dynamics subject to a constant control action, say $u=\bar{T}$, is studied for both models. Hence, the parameters $a_{i j}$ that appear in (17),(18) are denoted as $\bar{a}_{i j}=a_{i j}(\bar{T})$, while the same convention is adopted for those in (21)-(23).

\subsection{The $\mathrm{NO}_{2}$ gas sensor model: open-loop analysis}

It is immediate from $(17),(18)$ that, in open-loop configuration, i.e. a constant control action $u=\bar{T}$, the resulting system, namely

$$
\begin{aligned}
& \dot{x}_{1}=-\left(\bar{a}_{11}+\bar{a}_{12}\right) x_{1}-\bar{a}_{11} x_{2}+\bar{a}_{11} \mathrm{~S}_{0}, \\
& \dot{x}_{2}=-\left(\mu \bar{a}_{21}+\bar{a}_{22}\right) x_{2}+\mu \bar{a}_{21} \mathrm{~S}_{0},
\end{aligned}
$$

is affine.

Proposition 1. System $(27),(28)$ exhibits an asymptotically stable equilibrium point, which lies in $\mathbb{R}^{+} \times \mathbb{R}^{+}$.

Proof A straightforward calculation shows that the equilibrium solution, $x^{*}=\left(x_{1}^{*}, x_{2}^{*}\right)^{\top}$, is

$$
x_{1}^{*}=\frac{\bar{a}_{11} \bar{a}_{22} \mathrm{~S}_{0}}{\left(\bar{a}_{11}+\bar{a}_{12}\right)\left(\mu \bar{a}_{21}+\bar{a}_{22}\right)}, \quad x_{2}^{*}=\frac{\mu \bar{a}_{21} \mathrm{~S}_{0}}{\mu \bar{a}_{21}+\bar{a}_{22}},
$$

with $x_{1}^{*}, x_{2}^{*}>0$. In turn, the system matrix is

$$
\left(\begin{array}{cc}
-\left(\bar{a}_{11}+\bar{a}_{12}\right) & -\bar{a}_{11} \\
0 & -\left(\mu \bar{a}_{21}+\bar{a}_{22}\right)
\end{array}\right)
$$

with the diagonal elements coinciding with the eigenvalues, both lying in $\mathbb{R}^{-}$.

Proposition 2. In system (27),(28), the density of charged surface states in the equilibrium, $N_{s}^{*}=x_{1}^{*}+x_{2}^{*}$, increases as a function of the $\mathrm{NO}_{2}$ concentration, i.e. of $\mu$.

Proof In accordance with the proof of Proposition 1, $N_{s}^{*}$ can be written as:

$$
N_{s}^{*}=\frac{\left[\bar{a}_{11} \bar{a}_{22}+\mu \bar{a}_{21}\left(\bar{a}_{11}+\bar{a}_{12}\right)\right] \mathrm{S}_{0}}{\left(\bar{a}_{11}+\bar{a}_{12}\right)\left(\mu \bar{a}_{21}+\bar{a}_{22}\right)} ;
$$

Then, for all $\mu>0$,

$$
\frac{\partial N_{s}^{*}}{\partial \mu}=\frac{\bar{a}_{12} \bar{a}_{21} \bar{a}_{22} \mathrm{~S}_{0}}{\left(\bar{a}_{11}+\bar{a}_{12}\right)\left(\mu \bar{a}_{21}+\bar{a}_{22}\right)^{2}}>0 .
$$


4.2. The CO gas sensor model: open-loop analysis

Under an open-loop, constant control action $u=\bar{T}$, system (21)-(23) results in

$$
\begin{aligned}
& \dot{x}_{1}=\bar{a}_{11} e^{-\frac{\left(x_{1}-x_{2}+x_{3}\right)^{2}}{T}}\left(N_{i}^{\prime}-x_{1}\right)-\bar{a}_{12} x_{1}, \\
& \dot{x}_{2}=\bar{a}_{21}-\bar{a}_{22} e^{-\frac{\left(x_{1}-x_{2}+x_{3}\right)^{2}}{T}} x_{2}, \\
& \dot{x}_{3}=\bar{a}_{31} e^{-\frac{\left(x_{1}-x_{2}+x_{3}\right)^{2}}{T}}-\bar{a}_{32} x_{3},
\end{aligned}
$$

with $\bar{a}_{21}=\bar{a}_{21}(\mu), \bar{a}_{31}=\bar{a}_{31}(\mu)$.

The analysis of the equilibrium points of (29)-(31) requires the following Lemma:

Lemma 1. Let $F:=\left(0, N_{i}^{\prime}\right) \longrightarrow \mathbb{R}$ be defined as:

$$
F(z):=\beta_{1}\left(N_{i}^{\prime}-z\right) e^{-\frac{1}{T}\left(z-\frac{\beta_{2}\left(N_{i}^{\prime}-z\right)}{z}+\frac{\beta_{3} z}{N_{i}^{\prime}-z}\right)^{2}}-z,
$$

with

$$
\beta_{1}=\frac{\bar{a}_{11}}{\bar{a}_{12}}, \beta_{2}=\frac{\bar{a}_{11} \bar{a}_{21}}{\bar{a}_{12} \bar{a}_{22}}, \beta_{3}=\frac{\bar{a}_{12} \bar{a}_{31}}{\bar{a}_{11} \bar{a}_{32}} .
$$

If the equation $F(z)=0$ has a non degenerate solution in $\left(0, N_{i}^{\prime}\right)$, then it has at least another solution in such interval.

Proof Notice that

$$
\lim _{z \rightarrow 0^{+}} F(z)=0^{-}, \quad \lim _{z \rightarrow N_{i}^{\prime}} F(z)=-N_{i}^{\prime-} .
$$

Hence, the result follows immediately from the continuity of $F$ in $\left(0, N_{i}^{\prime}\right)$ and Bolzano's Theorem.

Proposition 3. Assume that the equation $F(z)=0$, with $F$ defined in (32), has a non degenerate solution in $\left(0, N_{i}^{\prime}\right)$. Then, system (29)-(31) has at least two equilibrium points, $x^{*}$, in $\left(0, N_{i}^{\prime}\right) \times \mathbb{R}^{+} \times \mathbb{R}^{+}$, with

$$
x_{1}^{*}=z^{*}, \quad x_{2}^{*}=\frac{\beta_{2}\left(N_{i}^{\prime}-z^{*}\right)}{z^{*}}, \quad x_{3}^{*}=\frac{\beta_{3} z^{*}}{N_{i}^{\prime}-z^{*}},
$$

where $\beta_{2,3}$ are defined in (33), and $z^{*} \in\left(0, N_{i}^{\prime}\right)$ verifying $F\left(z^{*}\right)=0$. 
Proof Algebraic manipulation shows that the $x_{1}$ component of the equilibrium points of (29)-(31), $x_{1}^{*}$, satisfies $F\left(x_{1}^{*}\right)=0$, while $x_{2,3}^{*}$ depend on $x_{1}^{*}$ as stated in (34). As $F\left(x_{1}^{*}\right)=0$ has one solution in $\left(0, N_{i}^{\prime}\right)$ by hypothesis, the result follows straightforwardly from Lemma 1.

Proposition 4. Let $x^{*}$ be an equilibrium point of system (29)-(31), with $x_{1}^{*} \in\left(0, N_{i}^{\prime}\right)$. If $N_{s}^{*}=x_{1}^{*}-x_{2}^{*}+x_{3}^{*}>0$, then $x^{*}$ is asymptotically stable.

Proof The Jacobian matrix of (29)-(31) at an equilibrium, $x^{*}$, can be written as

$$
J\left(x^{*}\right)=\gamma_{4}\left(\begin{array}{ccc}
-1-\gamma_{1} & 1 & -1 \\
1 & -1-\gamma_{2} & 1 \\
-1 & 1 & -1-\gamma_{3}
\end{array}\right)
$$

with

$$
\gamma_{1}=\frac{\bar{T} N_{i}^{\prime *}}{2 N_{s}^{*} x_{1}^{*}\left(N_{i}^{\prime}-x_{1}^{*}\right)}, \gamma_{2}=\frac{\bar{T}}{2 N_{s}^{*} x_{2}^{*}}, \gamma_{3}=\frac{\bar{T}}{2 N_{s}^{*} x_{3}^{*}}, \gamma_{4}=\frac{8 \alpha_{12} \alpha_{21} \alpha_{31} x_{1}^{*} x_{3}^{*}}{\bar{T}^{3}} .
$$

By hypothesis it is $\gamma_{i}>0$ and $J\left(x^{*}\right)$ is symmetric; hence, the result follows using Sylvester's criterion.

Remark 1. It has been numerically found out that, for temperature and $C O$ concentrations in the range of interest $\left(200^{\circ} \mathrm{C}<\mathrm{T}<400^{\circ} \mathrm{C}, 0<\mathrm{CO}<\right.$ $55 p p m$, system (29)-(31) presents two equilibrium points with $x_{1}^{*} \in\left(0, N_{i}^{\prime}\right)$. One of them is such that $N_{s}^{*}>0$, and hence asymptotically stable, while the other one is unstable.

\section{Sliding mode control-based analysis}

As detailed in Section 2, the control goal is to regulate the total density of charged surface states, $N_{s}$, of system (16) at a certain reference level, $N_{s}^{*}$, by using a temperature-based sigma-delta control loop acting as follows:

$$
u=\left\{\begin{array}{lll}
T_{B I T 0} & \text { if } \quad N_{s}>N_{s}^{*} \\
T_{B I T 1} & \text { if } \quad N_{s}<N_{s}^{*}
\end{array}\right.
$$

where $T_{B I T 0}, T_{B I T 1}$ are defined in (2),(3), and $N_{s}^{*}$ is a certain threshold value. From $(7),(8)$ we may see that $N_{s}$ is a linear combination of the state components, namely, $N_{s}=c^{\top} x$, with

$$
c^{\top}=(1,1) \text { and } c^{\top}=(1,-1,1)
$$


for the $\mathrm{NO}_{2}$ model (17),(18), and the $\mathrm{CO}$ model (21)-(23), respectively. Taking this into account, we define the switching function

$$
\sigma:=c^{\top} x-N_{s}^{*}
$$

Hence, the closed-loop dynamics of system (16) under the control law (35) within the time interval $\left[n T_{S},(n+1) T_{S}\right)$ is given by:

$$
\dot{x}=\frac{1+\operatorname{sign}\left(\sigma\left(n T_{S}\right)\right)}{2} f\left(x, T_{B I T 0}(t)\right)+\frac{1-\operatorname{sign}\left(\sigma\left(n T_{S}\right)\right)}{2} f\left(x, T_{B I T 1}(t)\right),
$$

with the vector fields defined as

$$
\begin{aligned}
& f\left(x, T_{B I T 0}(t)\right)=f_{l}(x) \cdot \mathbb{1}_{\left[n T_{S},(n+1-\delta) T_{S}\right)}(t)+f_{h}(x) \cdot \mathbb{1}_{\left[(n+1-\delta) T_{S},(n+1) T_{S}\right)}(t), \\
& f\left(x, T_{B I T 1}(t)\right)=f_{l}(x) \cdot \mathbb{1}_{\left[n T_{S},(n+\delta) T_{S}\right)}(t)+f_{h}(x) \cdot \mathbb{1}_{\left[(n+\delta) T_{S},(n+1) T_{S}\right)}(t),
\end{aligned}
$$

where

$$
f_{l}(x):=f\left(x, T_{l}, \mu\right), \quad f_{h}(x):=f\left(x, T_{h}, \mu\right) .
$$

The theoretical analysis will be carried on the average system (16),(35). The resulting dynamics is given in next proposition.

Proposition 5. When the sampling period, $T_{S}$, tends to zero, the averaged sigma-delta controlled system (16),(35) may be written as the switched system

$$
\dot{x}= \begin{cases}f_{0}(x) & \text { if } \quad \sigma>0, \\ f_{1}(x) & \text { if } \quad \sigma<0,\end{cases}
$$

with

$$
\begin{aligned}
& f_{0}(x)=(1-\delta) f_{l}(x)+\delta f_{h}(x), \\
& f_{1}(x)=\delta f_{l}(x)+(1-\delta) f_{h}(x) .
\end{aligned}
$$

Proof Straightforward following [35, 31].

The switching surface is attractive if solutions starting nearby the surface are directed towards it. A sufficient condition for this to happen is that $\sigma \dot{\sigma}<0$ [26], with $\dot{\sigma}$ computed from (36) over the system trajectories:

$$
\dot{\sigma}=c^{\top} \dot{x}=\left\{\begin{array}{lll}
c^{\top} f_{0}(x) & \text { if } & \sigma>0, \\
c^{\top} f_{1}(x) & \text { if } & \sigma<0 .
\end{array}\right.
$$


Hence, the attractive sliding region or sliding domain [26], $\Omega$, is given by

$$
\Omega:=\left\{x \in \mathbb{R}^{n}: c^{\top} f_{0}(x)<0 \wedge c^{\top} f_{1}(x)>0 \wedge \sigma(x)=0\right\} .
$$

Let us assume that (37) undergoes sliding motion over the switching surface, i.e. that the sliding domain is nonempty. Then, the ideal sliding dynamics on $\sigma=0$ described by Filippov's regularization [26] is a convex combination of the vector fields acting on the corresponding space region, $f_{0}, f_{1}$, namely,

$$
\dot{x}=\alpha(x) f_{0}(x)+\left.(1-\alpha(x)) f_{1}(x)\right|_{\sigma=0},
$$

with the coefficient $\alpha(x)$ selected so as to guarantee that the motion is tangent to the manifold $\sigma=0$ :

$$
\alpha(x)=\left.\frac{c^{\top} f_{1}(x)}{c^{\top}\left(f_{1}(x)-f_{0}(x)\right)}\right|_{\sigma=0} .
$$

Replacing (38),(39) in (42),(43), and (41) one gets the following result:

Proposition 6. System (37) slides along the switching surface $\sigma=0$ if the sliding domain,

$$
\Omega:=\left\{x \in \mathbb{R}^{n}: \delta<\left.\frac{c^{\top} f_{h}(x)}{c^{\top}\left(f_{h}(x)-f_{l}(x)\right)}\right|_{\sigma=0}<1-\delta\right\},
$$

is nonempty. In this case, the ideal sliding dynamics is given by:

$$
\dot{x}=f_{h}(x)+\left.\frac{c^{\top} f_{h}(x)}{c^{\top}\left(f_{h}(x)-f_{l}(x)\right)}\left(f_{h}(x)-f_{l}(x)\right)\right|_{\sigma=0} .
$$

Once at this point it is worth highlighting that when Model 2 is evolving on the switching surface $N_{s}=N_{s}^{*}$, the vector fields $f_{l}(x), f_{h}(x)$ defined from (21)-(23) are affine in the state variables, because the state-dependent exponential factors become constant. In turn, Model 1 is indeed affine (recall $(17),(18))$. Hence, on the switching surface these vector fields answer to:

$$
f_{l, h}(x)=A_{l, h} x+B_{l, h} .
$$


Consequently, the ideal sliding dynamics (45) can be written as:

$$
\begin{aligned}
& \dot{x}=A_{h} x+B_{h}+ \\
& +\frac{c^{\top}\left(A_{h} x+B_{h}\right)}{c^{\top}\left[\left(A_{h}-A_{l}\right) x+\left(B_{h}-B_{l}\right)\right]}\left[\left(A_{h}-A_{l}\right) x+\left(B_{h}-B_{l}\right)\right]
\end{aligned}
$$

for some suitable matrices $A_{h}, A_{l}$ and vectors $B_{h}, B_{l}$. The inequality defining the sliding domain in (44) reads as:

$$
\delta<\alpha(x)=\frac{c^{\top}\left(A_{h} x+B_{h}\right)}{c^{\top}\left[\left(A_{h}-A_{l}\right) x+\left(B_{h}-B_{l}\right)\right]}<1-\delta .
$$

Finally, notice that eventual equilibrium points of the ideal sliding dynamics (46) is within the sliding domain only if they fulfill restriction (47).

Remark 2. According to (44), the existence of sliding motion depends on $\delta$, while the ideal dynamics given in (45) by Filippov's convex combination approach are independent of $\delta$. Even more, it is immediate from (44) that, as $\delta$ increases, the sliding domain is reduced, which reduces control capability as well. And, in any case, one must have $\delta \in\left(0, \frac{1}{2}\right]$, otherwise no sliding motion can be enforced with this control logic.

5.1. The $\mathrm{NO}_{2}$ gas sensor model: closed-loop analysis

For Model 1, (17),(18) reads as

$$
A_{l, h}:=\left(\begin{array}{cc}
-\alpha_{11}^{l, h} & -\alpha_{12}^{l, h} \\
0 & -\alpha_{22}^{l, h}
\end{array}\right), \quad B_{l, h}:=\left(\begin{array}{c}
\beta_{1}^{l, h} \\
\beta_{2}^{l, h}
\end{array}\right),
$$

with

$\alpha_{11}^{l, h}=a_{11}^{l, h}+a_{12}^{l, h}, \alpha_{12}^{l, h}=a_{11}^{l, h}, \alpha_{22}^{l, h}=\mu a_{21}^{l, h}+a_{22}^{l, h}, \beta_{1}^{l, h}=a_{11}^{l, h} S_{0}, \beta_{2}^{l, h}=\mu a_{21}^{l, h} S_{0}$, and $a_{i j}^{l, h}:=a_{i j}\left(T_{l, h}\right)$ in $(19),(20)$. Hence, the ideal sliding dynamics is given by:

$$
\begin{aligned}
& x_{1}=N_{s}^{*}-x_{2}, \\
& \dot{x}_{2}=-\alpha_{22}^{h} x_{2}+\beta_{2}^{h}+\alpha(x)\left[\left(\alpha_{22}^{l}-\alpha_{22}^{h}\right) x_{2}+\beta_{2}^{h}-\beta_{2}^{l}\right],
\end{aligned}
$$

with

$$
\alpha(x)=\alpha\left(x_{2}\right)=\frac{\eta_{0} x_{2}+\eta_{1}}{\eta_{2} x_{2}+\eta_{3}}
$$


where

$$
\begin{gathered}
\eta_{0}=\alpha_{11}^{h}-\alpha_{12}^{h}-\alpha_{22}^{h}, \quad \eta_{1}=\beta_{1}^{h}-\beta_{2}^{h}-\alpha_{11}^{h} N_{s}^{*} \\
\eta_{2}=\alpha_{11}^{h}-\alpha_{11}^{l}+\alpha_{12}^{l}-\alpha_{12}^{h}, \quad \eta_{3}=\left(\alpha_{11}^{l}-\alpha_{11}^{h}\right) N_{s}^{*}+\beta_{2}^{h}-\beta_{2}^{l} .
\end{gathered}
$$

Then:

Proposition 7. The ideal sliding dynamics (48),(49) has, at most, two equilibrium points. Moreover, assume that $x^{*}=\left(x_{1}^{*}, x_{2}^{*}\right)^{\top}$ is an equilibrium of (48),(49):

(i) If

$$
\delta<\frac{\alpha_{22}^{h} x_{2}^{*}-\beta_{2}^{h}}{\left(\alpha_{22}^{l}-\alpha_{22}^{h}\right) x_{2}^{*}+\beta_{2}^{h}-\beta_{2}^{l}}<1-\delta,
$$

then the equilibrium belongs to the sliding domain.

(ii) Let

$$
h\left(x_{2}^{*}\right)=-\alpha_{22}^{h}+\left(\alpha_{22}^{l}-\alpha_{22}^{h}\right) \alpha\left(x_{2}^{*}\right)+\frac{\eta_{0} \eta_{3}-\eta_{1} \eta_{2}}{\left(\eta_{2} x_{2}^{*}+\eta_{3}\right)^{2}}
$$

if $h\left(x_{2}^{*}\right)<0$ then $x^{*}$ is asymptotically stable.

Proof The first statement follows from the fact that the search of equilibria in (49) yields a quadratic equation in $x_{2}^{*}$. In turn, (i) follows after a straightforward algebraic manipulation, and (ii) is a result of computing the Jacobian of (49) at $x_{2}^{*}$ and analyzing the equilibrium character of $x_{2}^{*}$.

\subsection{The CO gas sensor model: closed-loop analysis}

For Model 2, (21)-(23) become:

$$
A_{l, h}:=-\operatorname{diag}\left(\alpha_{1}^{l, h}, \alpha_{2}^{l, h}, \alpha_{3}^{l, h}\right), B_{l, h}:=\left(\begin{array}{c}
\beta_{1}^{l, h} \\
\beta_{2}^{l, h}
\end{array}\right),
$$

with

$$
\begin{aligned}
& \alpha_{1}^{l, h}=a_{11}^{l, h} e^{-\frac{N_{s}^{*}}{T_{l, h}}}, \alpha_{2}^{l, h}=\frac{a_{22}^{l, h} \alpha_{1}^{l, h}}{a_{11}^{l, h}}, \alpha_{3}^{l, h}=a_{32}^{l, h}, \\
& \beta_{1}^{l, h}=\alpha_{1}^{l, h} N_{i}^{\prime}, \quad \beta_{2}^{l, h}=a_{22}^{l, h}, \quad \beta_{3}^{l, h}=a_{31}^{l, h} e^{-\frac{N_{s}^{*}}{T_{l, h}}}
\end{aligned}
$$


and $a_{i j}^{l, h}:=a_{i j}\left(T_{l, h}\right)$ in (24)-(26). Hence, the ideal sliding dynamics is given by:

$$
\begin{aligned}
& x_{1}=N_{s}^{*}+x_{2}-x_{3}, \\
& \dot{x}_{i}=-\alpha_{i}^{h} x_{i}+\beta_{i}^{h}+\alpha(x)\left[\left(\alpha_{i}^{l}-\alpha_{i}^{h}\right) x_{i}+\beta_{i}^{h}-\beta_{i}^{l}\right],
\end{aligned}
$$

with $i=2,3$ and

$$
\alpha(x)=\alpha\left(x_{2}, x_{3}\right)=\frac{\eta_{0} x_{2}+\eta_{1} x_{3}+\eta_{2}}{\eta_{3} x_{2}+\eta_{4} x_{3}+\eta_{5}},
$$

where

$$
\begin{aligned}
& \eta_{0}=\alpha_{1}^{h}-\alpha_{2}^{h}, \eta_{1}=\alpha_{3}^{h}-\alpha_{1}^{h}, \eta_{2}=\beta_{1}^{h}-\beta_{2}^{h}+\beta_{3}^{h}+\alpha_{1}^{h} N_{s}^{*}, \eta_{3}=\alpha_{1}^{h}-\alpha_{1}^{l}+\alpha_{2}^{l}-\alpha_{2}^{h}, \\
& \eta_{4}=\alpha_{3}^{h}-\alpha_{3}^{l}+\alpha_{1}^{l}-\alpha_{1}^{h}, \eta_{5}=\beta_{1}^{h}-\beta_{1}^{l}+\beta_{2}^{l}-\beta_{1}^{h}+\beta_{3}^{h}-\beta_{3}^{l}+\left(\alpha_{1}^{h}-\alpha_{1}^{l}\right) N_{s}^{*} .
\end{aligned}
$$

Then:

Proposition 8. The ideal sliding dynamics (50),(51) has, at least, one real equilibrium, and at most, three. Moreover, assume that $x^{*}=\left(x_{1}^{*}, x_{2}^{*}, x_{3}^{*}\right)^{\top}$ is an equilibrium of $(50),(51)$ :

(i) If

$$
\delta<\frac{\alpha_{3}^{h} x_{2}^{*}-\beta_{3}^{h}}{\left(\alpha_{3}^{l}-\alpha_{3}^{h}\right) x_{3}^{*}+\beta_{2}^{h}-\beta_{2}^{l}}<1-\delta,
$$

then the equilibrium belongs to the sliding domain.

(ii) If the eigenvalues of the Jacobian matrix of system (51) evaluated at $\left(x_{2}^{*}, x_{3}^{*}\right)$ lie in the left complex plane, the equilibrium $x^{*}$ is asymptotically stable.

Proof The first statement follows from the fact that the search of equilibria in (51) yields

$$
x_{2}^{*}=\frac{\left(\alpha_{3}^{h} \beta_{2}^{l}-\alpha_{3}^{l} \beta_{2}^{h}\right) x_{3}^{*}+\beta_{2}^{h} \beta_{3}^{l}-\beta_{2}^{l} \beta_{3}^{h}}{\left(\alpha_{2}^{l} \alpha_{3}^{h}-\alpha_{2}^{h} \alpha_{3}^{l}\right) x_{3}^{*}+\alpha_{2}^{l} \beta_{3}^{h}-\alpha_{2}^{h} \beta_{3}^{l}},
$$

and, consequently, a cubic equation in $x_{3}^{*}$. In turn, (i) follows after a straightforward algebraic manipulation, and (ii) stems from well-known stability results. 


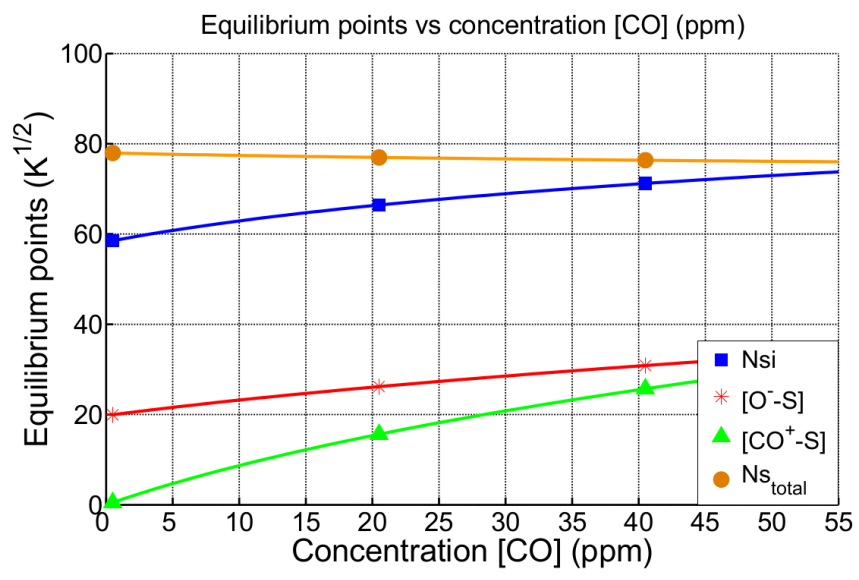

Figure 3: Open-loop stable equilibrium points as a function of the $[\mathrm{CO}]$ concentration. Temperature: $T=250^{\circ} \mathrm{C}$.

\section{Numerical results}

\subsection{Open-loop and closed-loop analysis}

In this subsection we mainly focus on Model 2 because it is a non-linear model, and hence it shows much richer dynamics than Model 1, which is linear.

The first objective of the simulations is to compare the evolution of the equilibrium concentrations for different values of $\mu=[\mathrm{CO}]$, when the sensor is in open or closed loop configuration. Figure 3 shows how the equilibrium points $\left(N_{s i}^{*},\left[\mathrm{CO}^{+}-\mathrm{S}\right]^{*},\left[\mathrm{O}^{-}-\mathrm{S}\right]^{*}\right)$ change in the open loop case. Figure 4 shows the equivalent result under closed loop control (for a chosen value $N_{s}^{*}=$ $\left.82 K^{1 / 2}\right)$. The control action drives the system to the closed-loop equilibrium points, which are those calculated with the analytical expression of the sliding dynamics (42). By comparing Figures 3 and 4, we see that the excursion of the equilibrium points for the selected range of [CO] concentrations is smaller when the system is in closed loop.

The second objective of this subsection is to observe how changes in the main operating parameters of the control loop, namely $T_{h}$ and $\delta$, affect the size of the attractive sliding region. Figure 5 shows the maximum and minimum $[\mathrm{CO}]$ concentrations so that the equilibrium points lie in the attractive 


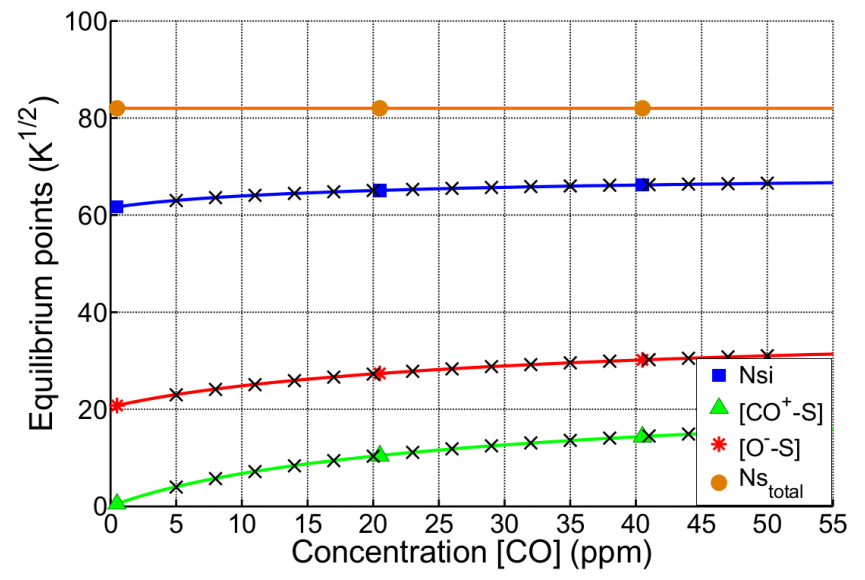

Figure 4: Closed-loop attractive equilibrium points as a function of the $\mu=[\mathrm{CO}]$ concentration. Actuation temperatures: $T_{l}=200^{\circ} \mathrm{C}, T_{h}=350^{\circ} \mathrm{C} ; \delta=0.1$. The crosses represent the equilibrium points calculated executing closed loop simulations, while the lines corresponds to the equilibrium points calculated with the analytical expression of the ideal sliding dynamics (42). Each line represents a state variable.

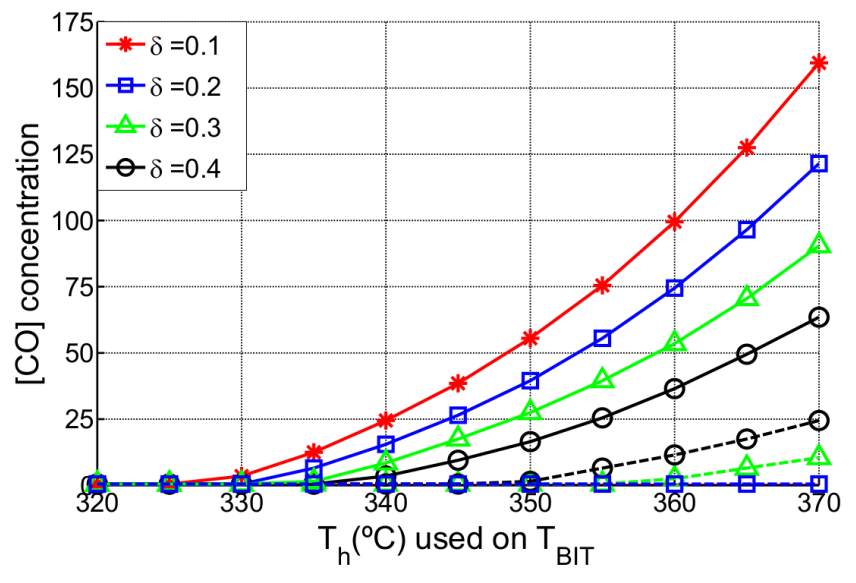

Figure 5: Maximum and minimum [CO] concentration curves as a function of $T_{h}$ for $\delta$ varying between 0.1 and $0.4, N_{s}^{*}=82 K^{1 / 2}$. The dotted curves correspond to the minimum values. 


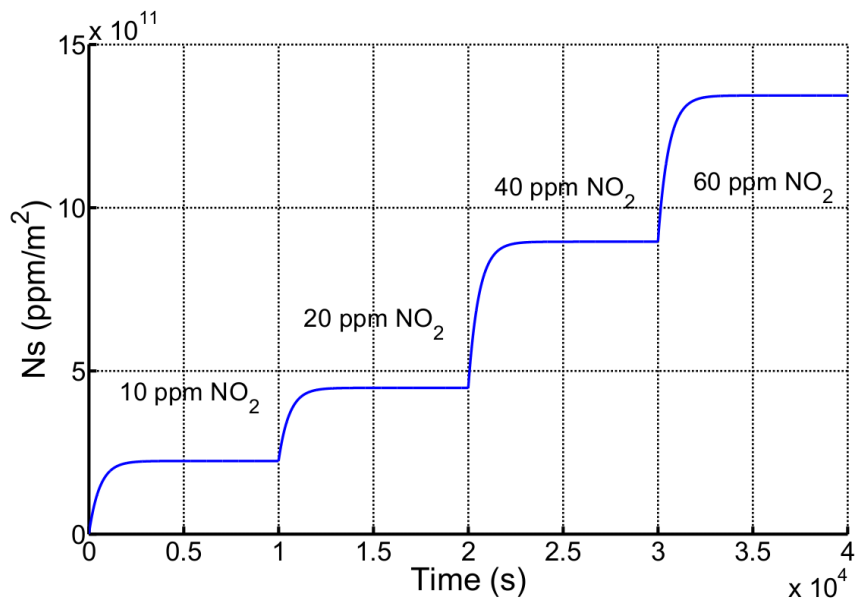

Figure 6: Total surface density of states response in open loop system, applying a fixed temperature $T=350^{\circ} \mathrm{C}$. Time evolution for different concentrations of $\mathrm{NO}_{2}: 10,20,40$, $60 \mathrm{ppm}$.

sliding region. These values obviously depend on $\delta$, as highlighted in Remark 2. For example, when $\delta=0.3$, the green curves show the minimum and maximum $[\mathrm{CO}]$ concentration, as a function of $T_{h}$, so that the system can be effectively controlled, i.e., the equilibrium points lie in the attractive region. Reducing the value of $\delta$, or increasing $T_{h}$, results in an improvement of the acceptable range of $[\mathrm{CO}]$ concentrations, which is something that may be expected.

\subsection{Transient responses due to changes in gas concentration}

The goal now is to study the transient responses in the system to changes in the external gas concentration $\left(\left[\mathrm{NO}_{2}\right]\right.$ for Model 1 and $\left[\mathrm{CO}_{2}\right]$ for Model 2). A comparison is made between open and closed-loop responses.

\subsubsection{Model 1: $\mathrm{NO}_{2}$}

Figure 6 shows the transients of the open-loop response of the total density of charged states, $N_{s}$, to different concentrations of $\mathrm{NO}_{2}$ at constant temperature, $T_{h}=350^{\circ} \mathrm{C}$. The time response in this case is approximately 40 minutes. As predicted by Proposition 2, higher values of $\mathrm{NO}_{2}$ concentration increase the total number of ionized states. 


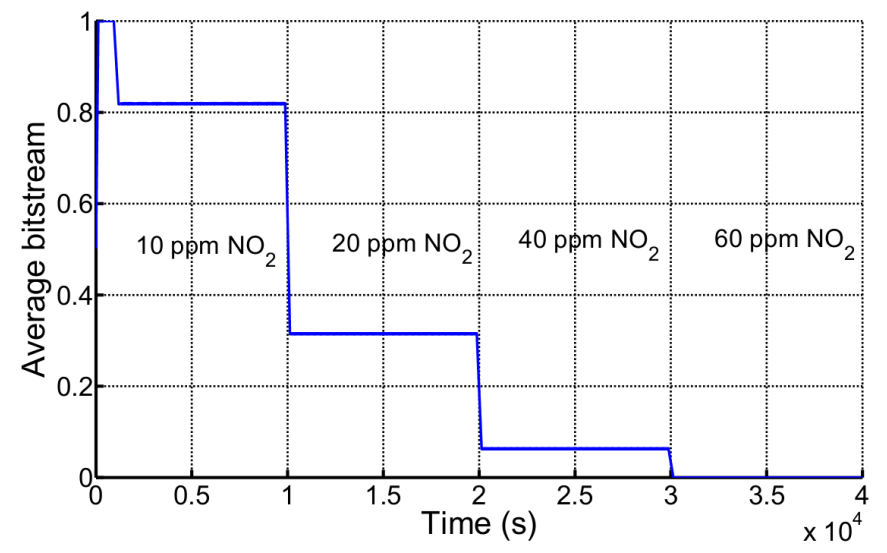

Figure 7: Average bitstream of the closed-loop system response. Time evolution for different concentrations of $\mathrm{NO}_{2}: 10,20,40,60 \mathrm{ppm}$. Saturated response for $60 \mathrm{ppm}$ of $\mathrm{NO}_{2}$ (last time interval). $T_{l}=250^{\circ} \mathrm{C}, T_{h}=400^{\circ} \mathrm{C}$.

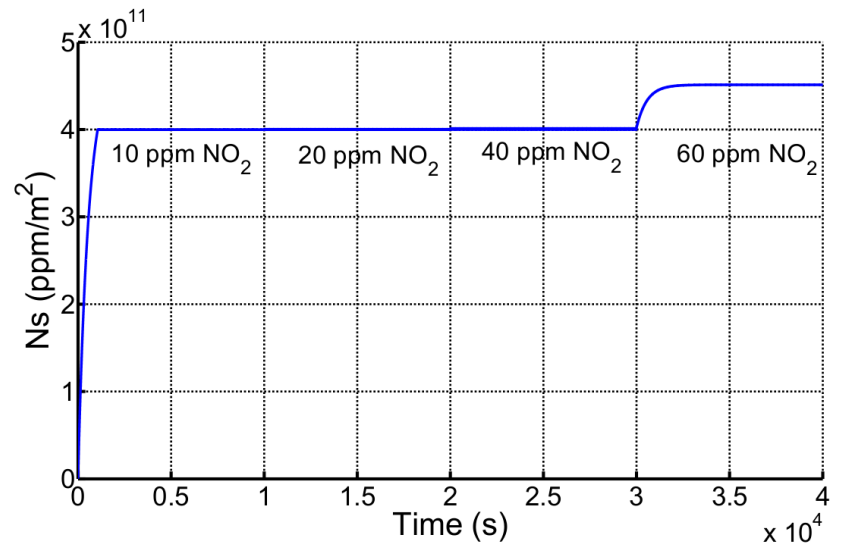

Figure 8: Surface density of states response in close loop system. Time evolution for different concentrations of $\mathrm{NO}_{2}: 10,20,40,60 \mathrm{ppm}$. Saturated response for $60 \mathrm{ppm}$ of $\mathrm{NO}_{2}$ (last time interval). $T_{l}=250^{\circ} \mathrm{C}, T_{h}=400^{\circ} \mathrm{C}$.

Figure 7, on the other hand, shows the result of applying the control technique to the system. In this figure, the depicted average bitstream is 


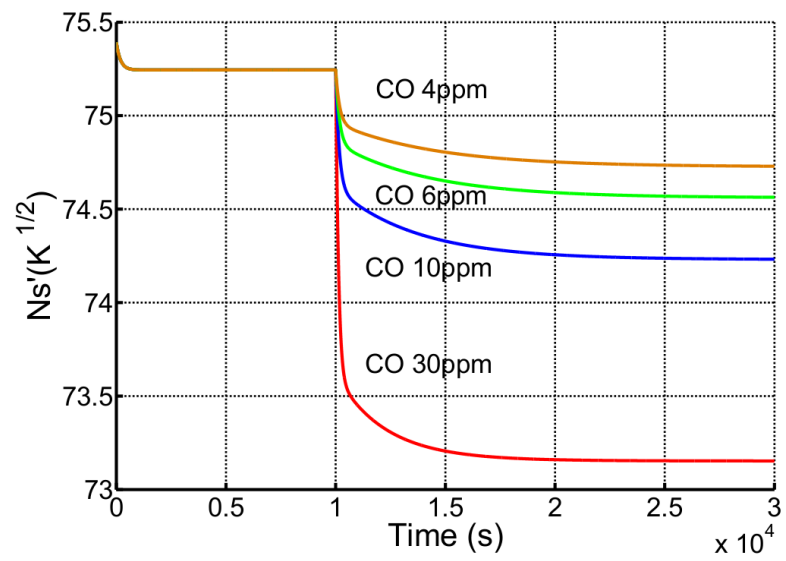

Figure 9: Total surface density of states response in open loop configuration, applying a fixed temperature $T=200^{\circ} \mathrm{C}$. Two time intervals: a) From 0 to $10000 \mathrm{~s}$, without CO. b) From 10000s to 30000 s, with CO. Different curves for different concentrations of CO: 30 , 10, 6, 4 ppm.

defined as the simple moving average of the ' 0 ' and ' 1 ' bit values taken when $T_{\mathrm{BIT} 0}$ and $T_{\mathrm{BIT} 1}$ are respectively used in the control loop during the simulation. As it can be observed the time response has been improved.

The average bitstream (directly related to the average temperature in the sensor) decreases for increasing gas concentrations. For the maximum concentration in the simulation, $60 \mathrm{ppm}$, the control saturates, because the average bitstream goes down to 0. This fact is also reflected in Figure 8, which shows the time evolution of the total density of charged species, $N_{s}$, as a function of time. For the first three concentrations, the control action is able to keep $N_{s}$ constant at $N_{s}^{*}=4 \cdot 10^{11} \mathrm{ppm} / \mathrm{m}^{2}$, thus illustrating the robustness of the design to parametric disturbances. Once the control saturates, $N_{s}$ varies with time, as it may be expected.

\subsubsection{Model 2: $C O$}

In this section, we present the simulation results of the model described in Section 3.2. Figure 9 shows the evolution of the total surface density of states when the sensor is in open loop for different values of $\mu=[\mathrm{CO}]$ concentrations. The time response is approximately $200 \mathrm{~min}$ in this case. For higher $\mathrm{CO}$ concentrations, $N_{s}$ decreases. This is to be expected, since CO is a reducing 
species. On the other hand, Figure 10 shows the equivalent transients, for the same $[\mathrm{CO}]$ concentration changes, under closed loop operation. The observed time response has been improved with respect to the open-loop response, but not as much as in the $\mathrm{NO}_{2}$ case.

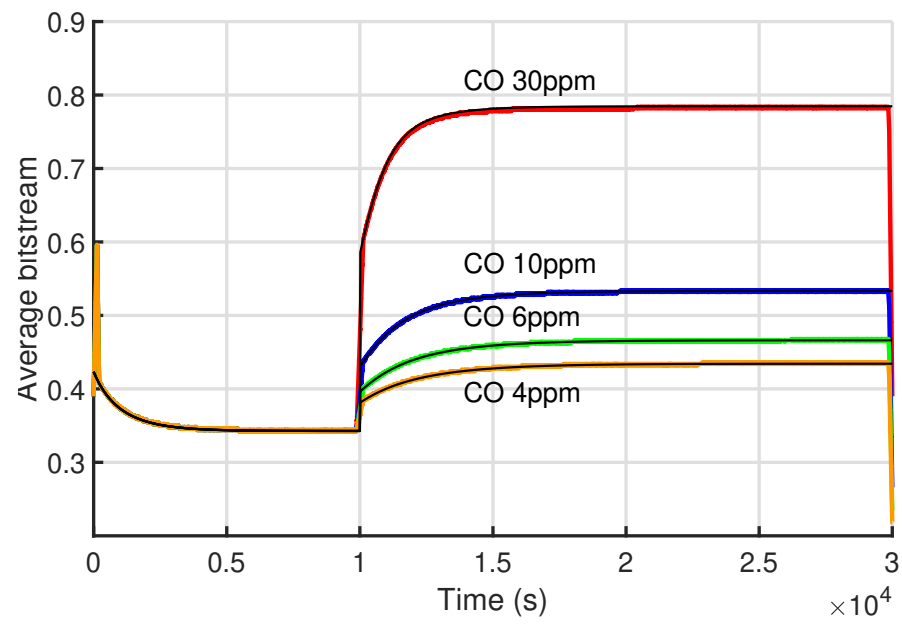

Figure 10: Average bitstream of the closed-loop system response. Two time intervals: a) From 0 to 10000s, without CO. b) From 10000s to 30000s, with CO. Different curves for different concentrations of CO: 30, 10, 6, 4 ppm. Colored lines: simulation results, black lines: analytical results. $T_{l}=200^{\circ} \mathrm{C}, T_{h}=350^{\circ} \mathrm{C}$.

Figure 11 shows a comparison between the simulation results obtained for the closed-loop system and those given by the ideal analytical expression of the average bitstream of the sigma-delta control (43). As it can be observed, there is a good match between the analytical prediction (black lines) and the simulations (colored lines).

Finally, Figure 12 shows the frequency spectrum of the output bitstream. This simulation shows a slope of $20 \mathrm{~dB} / \mathrm{dec}$, which corresponds to the expected sigma-delta quantization noise-shaping (a first order zero at null frequency). This is a remarkable feature, and a signature of the fact that the system is a switched affine system under constant surface potential operation.

\section{Conclusions}

An analysis of the dynamics of MOX gas sensors under a sigma-delta control of the surface potential has been presented. Using state space models, 


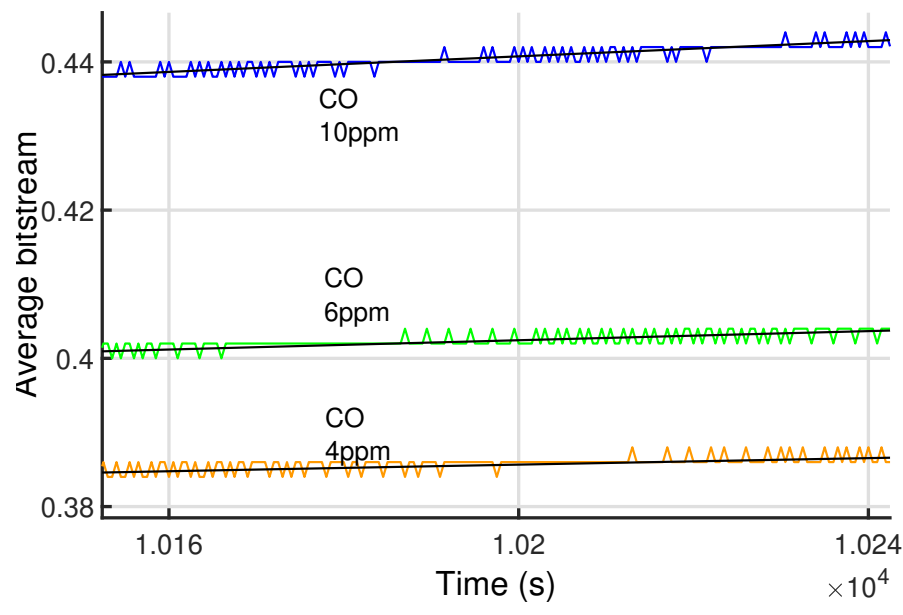

Figure 11: Zoom of the average bitstream (Figure 10). Colored lines: numerical results, black lines: analytical results.

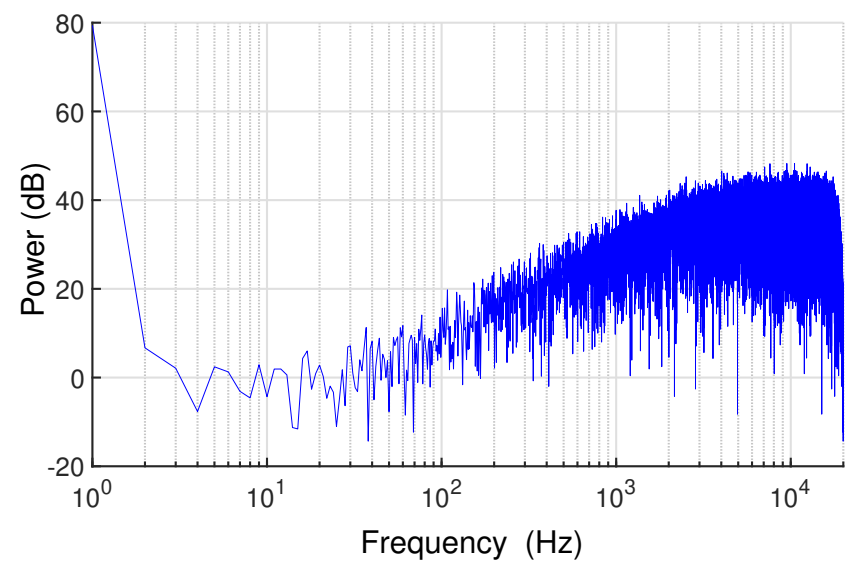

Figure 12: Frequency response of the stabilized closed loop system. Numerical results show a slope of $20 \mathrm{~dB} / \mathrm{dec}$.

it has been possible to study the time evolution of the chemical species within the sensing layer, namely the adsorbed and ionized states. In the infinite sampling frequency approximation the system can be analyzed as a sliding mode controller in which the control surface is equivalent to the condition: constant surface potential. The equilibrium points in open and closed loop 
configurations have been analyzed. Results have been provided about the existence of an attractive sliding region. Finally, numerical results have been presented to observe specific working examples and validate the analytical predictions.

\section{Acknowledgements}

O. Monge and M. Dominguez-Pumar were supported in part by the Spanish Ministry MINECO under Projects ESP2016-79612-C3-2-R and TEC201348102-C2-1P. J.M. Olm was partially supported by the Spanish MINECO project DPI2017-85404-P.

[1] S. Capone, M. Zuppa, D. S. Presicce, L. Francioso, F. Casino, P. Siciliano, Metal oxide gas sensor array for the detection of diesel fuel in engine oil, Sensors and Actuators B: Chemical 131 (1) (2008) 125-133.

[2] M. Fleischer, Advances in application potential of adsorptive-type solid state gas sensors: high-temperature semiconducting oxides and ambient temperature GasFET devices, Measurement Science and Technology 19 (4) (2008) 042001.

[3] K. T. Ng, F. Boussaid, A. Bermak, A CMOS single-chip gas recognition circuit for metal oxide gas sensor arrays, IEEE Transactions on Circuits and Systems I: Regular Papers 58 (7) (2011) 1569-1580.

[4] G. Sberveglieri, Recent developments in semiconducting thin-film gas sensors, Sensors and Actuators B: Chemical 23 (2-3) (1995) 103-109.

[5] G. Korotcenkov, Metal oxides for solid-state gas sensors: What determines our choice?, Materials Science and Engineering: B 139 (1) (2007) $1-23$.

[6] J. Cheng, J. Wang, Q. Li, H. Liu, Y. Li, A review of recent developments in tin dioxide composites for gas sensing application, Journal of Industrial and Engineering Chemistry 44 (Supplement C) (2016) $1-22$.

[7] C. Wang, L. Yin, L. Zhang, D. Xiang, R. Gao, Metal oxide gas sensors: sensitivity and influencing factors, Sensors 10 (3) (2010) 2088-2106. 
[8] G. Korotcenkov, Gas response control through structural and chemical modification of metal oxide films: state of the art and approaches, Sensors and Actuators B: Chemical 107 (1) (2005) 209-232.

[9] S. Vallejos, P. Umek, T. Stoycheva, F. Annanouch, E. Llobet, X. Correig, P. De Marco, C. Bittencourt, C. Blackman, Single-step deposition of au- and pt-nanoparticle-functionalized tungsten oxide nanoneedles synthesized via aerosol-assisted cvd, and used for fabrication of selective gas microsensor arrays, Advanced Functional Materials 23 (10) (2013) 1313-1322.

[10] N. M. Vuong, D. Kim, H. Kim, Surface gas sensing kinetics of a $\mathrm{WO}_{3}$ nanowire sensor: part 1-oxidizing gases, Sensors and Actuators B: Chemical 220 (2015) 932-941.

[11] J. Ding, T. J. McAvoy, R. E. Cavicchi, S. Semancik, Surface state trapping models for $\mathrm{SnO}_{2}$-based microhotplate sensors, Sensors and Actuators B: Chemical 77 (3) (2001) 597-613.

[12] S. Nakata, K. Neya, K. Takemura, Non-linear dynamic responses of a semiconductor gas sensor - competition effect on the sensor responses to gaseous mixtures, Thin Solid Films 391 (2) (2001) 293-298.

[13] G. Tulzer, S. Baumgartner, E. Brunet, G. C. Mutinati, S. Steinhauer, A. Köck, C. Heitzinger, Modeling $\mathrm{H}_{2}$ Adsorption Processes at $\mathrm{SnO}_{2}$ Nanowire Surfaces-Parameter Estimation and Simulation., in: BIODEVICES, 2013, pp. 265-268.

[14] B. Chwieroth, B. R. Patton, Y. Wang, Conduction and Gas-Surface Reaction Modeling in Metal Oxide Gas Sensors, Journal of Electroceramics 6 (1) (2001) 27-41.

[15] G. Tulzer, S. Baumgartner, E. Brunet, G. Mutinati, S. Steinhauer, A. Kšck, C. Heitzinger, Inverse modeling of co reactions at $\mathrm{SnO}_{2}$ nanowire surfaces for selective detection, Procedia Engineering 47 (2012) 809-812.

[16] G. Tulzer, S. Baumgartner, E. Brunet, G. C. Mutinati, S. Steinhauer, A. Köck, P. E. Barbano, C. Heitzinger, Kinetic parameter estimation and fluctuation analysis of co at $\mathrm{SnO}_{2}$ single nanowires, Nanotechnology 24 (31) (2013) 315501. 
[17] N. Barsan, U. Weimar, Conduction model of metal oxide gas sensors, Journal of Electroceramics 7 (3) (2001) 143-167.

[18] A. Pardo, S. Marco, J. Samitier, Nonlinear inverse dynamic models of gas sensing systems based on chemical sensor arrays for quantitative measurements, IEEE Transactions on Instrumentation and Measurement 47 (3) (1998) 644-651.

[19] J. Fonollosa, S. Sheik, R. Huerta, S. Marco, Reservoir computing compensates slow response of chemosensor arrays exposed to fast varying gas concentrations in continuous monitoring, Sensors and Actuators B: Chemical 215 (2015) 618-629.

[20] E. Llobet, R. Ionescu, S. Al-Khalifa, J. Brezmes, X. Vilanova, X. Correig, N. Bârsan, J. W. Gardner, Multicomponent gas mixture analysis using a single tin oxide sensor and dynamic pattern recognition, IEEE Sensors Journal 1 (3) (2001) 207-213.

[21] M.-S. Yao, W.-X. Tang, G.-E. Wang, B. Nath, G. Xu, MOF thin filmcoated metal oxide nanowire array: Significantly Improved Chemiresistor Sensor Performance, Advanced Materials 28 (26) (2016) 5229-5234.

[22] S. Wang, J. Yang, H. Zhang, Y. Wang, X. Gao, L. Wang, Z. Zhu, Onepot synthesis of $3 \mathrm{D}$ hierarchical $\mathrm{SnO}_{2}$ nanostructures and their application for gas sensor, Sensors and Actuators B: Chemical 207.

[23] J. Zhang, Y.-L. Luo, F. Xu, Y.-S. Chen, Novel poly(N-vinylcarbazole) grafted multi-walled carbon nanotube nanocomposites based on a nucleophilic reaction: Synthesis, fabrication of thin films, and sensing properties, Chemical Engineering Journal 298 (2016) 136-145.

[24] M. Dominguez-Pumar, L. Kowalski, R. Calavia, E. Llobet, Smart control of chemical gas sensors for the reduction of their time response, Sensors and Actuators B: Chemical 229 (2016) 1-6.

[25] M. Dominguez-Pumar, L. Kowalski, E. Llobet, R. Calavia, Active control of the surface potential of nanostructured layers, IEEE Sensors Journal 16 (8) (2016) 2213-2214.

[26] V. I. Utkin, Sliding modes in control and optimization, Springer-Verlag, 1992. 
[27] C.-T. Chen, S.-T. Peng, Nonlinear extendesign of a sliding mode control system for chemical processes, Journal of Process Control 15 (2005) 515530 .

[28] M. von Stosch, R. Oliveira, J. Peres, S. Feyo de Azevedo, Hybrid semiparametric modeling in process systems engineering: Past, present and future, Computers and Chemical Engineering 60 (2014) 86-101.

[29] G. Lara-Cisneros, R. Aguilar-López, R. Femat, On the dynamic optimization of methane production in anaerobic digestion via extremumseeking control approach, Computers and Chemical Engineering 75 (2015) 49-59.

[30] H. Sira-Ramírez, Sliding mode control: the delta-sigma modulation approach, Birkhäuser, 2015.

[31] M. Dominguez-Pumar, S. Gorreta, J. Pons-Nin, Sliding-mode analysis of the dynamics of sigma-delta controls of dielectric charging, IEEE Transactions on Industrial Electronics 63 (4) (2016) 2320-2329.

[32] N. Barsan, C. Simion, T. Heine, S. Pokhrel, U. Weimar, Modeling of sensing and transduction for p-type semiconducting metal oxide based gas sensors, Journal of Electroceramics 25 (1) (2010) 11-19.

[33] R. Ionescu, E. Llobet, S. Al-Khalifa, J. Gardner, X. Vilanova, J. Brezmes, X. Correig, Response model for thermally modulated tin oxide-based microhotplate gas sensors, Sensors and Actuators B: Chemical 95 (1) (2003) 203-211.

[34] A. Fort, M. Mugnaini, S. Rocchi, V. Vignoli, E. Comini, G. Faglia, A. Ponzoni, Metal-oxide nanowire sensors for $\mathrm{CO}$ detection: Characterization and modeling, Sensors and Actuators B: Chemical 148 (1) (2010) 283-291.

[35] H. Sira-Ramirez, M. Zribi, S. Ahmad, Pulse width modulated control of robotic manipulators, International Journal of Systems Science 24 (1993) 1423-1437. 